\title{
The Most Negative and Most Positive Expectation Values of the Spin Operator
}

\author{
Larry Zamick \\ Department of Physics and Astronomy,Rutgers University, \\ Piscataway,New Jersey,USA 08854* and \\ Weizmann Institute of Science, Rehovot, Israel
}

\begin{abstract}
Formulas for the most positive and most negative values of the expectation of the spin operator are given and compared with single particle values. The Nilsson model is used to evaluate these expectations and a scenario is discussed where the value is greater than one.
\end{abstract}

${ }^{*}$ Permanent Address 
The motivation for this work stems from the fact that isoscalar magnetic moments obtained from mirror pairs and $N=Z$ odd-odd nuclei have values which are very close to the single $j$ limit-simplicity in the midst of complexity. We wish to clarify the distinction between one particle and the many particle aspects of this problem.

For a system of several nucleons we define the expectation value of the spin operator $\vec{\sigma}=2 \vec{S}$

$$
\langle\sigma\rangle=\left\langle\Psi_{J}^{J} \sigma_{z} \Psi_{J}^{J}\right\rangle
$$

where $\Psi$ is the many-particle wave function in a state with $M=J$. The magnetic moment of a single nucleon in a state $\psi_{j}^{j}$ is called the Schmidt moment. From values of this moment we can infer that for a single nucleon in a state $[L, 1 / 2]^{j}$ with $j=L+1 / 2$ the value of $\langle\sigma\rangle$ is one; for a single nucleon with $j=L-1 / 2$ the value is $-j /(j+1)$. We next consider a system many nucleons and use $L S$ wave functions $[L, S] J$. We address the problem of what are the most negative and most positive values of $\langle\sigma\rangle$. We find

$$
\langle\sigma\rangle=(1 J 0 J \mid J J) \times 2 S /(1 S 0 S \mid S S) \sqrt{(2 J+1) \times(2 S+1)} W(1 S J L ; S J)
$$

where $\mathrm{W}$ is a Racah coefficient. We have

$$
(1 J 0 J \mid J J)=-\sqrt{J /(J+1)} \quad(1 S 0 S \mid S S)=-\sqrt{S /(S+1)}
$$

and

$$
W=-[S(S+1)+J(J+1)-L(L+1)] / \sqrt{4 S(S+1)(2 S+1) J(J+1)(2 J+1)}
$$

We find

$$
\langle\sigma\rangle=[S(S+1)+J(J+1)-L(L+1)] /(J+1) .
$$

Let us consider the extremes. For

$$
J=L-S \quad\langle\sigma\rangle=-2 S J /(J+1)
$$

This is the most negative value this quantity can have for a given $J$. This expression for several nucleons in $L S$ coupling with $J=L-S$ is consistent with the expression for a single nucleon with $j=L-1 / 2(-j /(j+1))$, as it must be. The maximum value of $\langle\sigma\rangle$ is obtained 
by setting $J=L+S$. The value is $2 S$. For a single nucleon the value is one. One can determine $\langle\sigma\rangle$ from mirror pairs:

$$
\langle\sigma\rangle=(2 \mu(I S)-J) /\left(\mu_{p}+\mu_{n}-1 / 2\right)
$$

where

$$
\mu(I S)=\left(\mu\left(T_{z}\right)+\mu\left(-T_{z}\right)\right) / 2
$$

In a work of Kramer et al.[1] the magnetic moment of ${ }^{21} \mathrm{Mg}$ is measured, which when combined with the moment of ${ }^{21} \mathrm{~F}$ yields an isoscalar magnetic moment and an expectation value of the spin operator. These authors refer to the "empirical limits" .They use as limits the single particle Schmidt values $-j /(j+1)$ for $j=L-1 / 2$ and one for $j=L+1 / 2$ and call the results beyond these limits anomalous. By this criterion their own value $\langle\sigma\rangle=1.15(2)$ is anomalous. They also refer to anomalies for $A=9$ found by Matsuta et al.[2] and discussed by Utsuno[3]. They obtained a very large value $\langle\sigma\rangle=1.44$. A careful reading of the Matsuta et al.and Kramer et al. papers however shows that they do not say that these empirical limits are theoretical limits. Indeed in ref[1] the authors report a shell model calculation with a charge independent interaction which gives a value 1.11, close to their measured value. They then go on to include a charge symmetry violating interaction which improves the fit . The final result is 1.15 . Their shell model calculation shows that one does not need a violation of charge symmetry to go beyond the "empirical limit" $\langle\sigma\rangle=1$.

We would say that their results are not anomalous if the theoretical limits are used. For $J=5 / 2$ an $L S$ wave function component with $L=0 S=5 / 2$ would yield an upper limit of five-much larger than the Schmidt limit of one. For $L=1 S=3 / 2$ we get three. It would be correct to say that these configurations are not the major components of the complete nuclear wave function so it is still surprising that values greater than one are obtained. For $A=9 J=3 / 2$ there are several $L S$ configurations with $\langle\sigma\rangle$ greater than one. For example there is $[311] L=0 T=3 / 2 S=3 / 2$ for which $\langle\sigma\rangle=3$, and $[221] L=1 T=3 / 2 S=3 / 2$ for which $\langle\sigma\rangle$ is $11 / 5[4]$. Note that the supermultiplet quantum numbers at the left in these two examples are not needed to evaluate $\langle\sigma\rangle$-only $L$ and $S$ are needed. However they are included to show that these states obey the Pauli principle. Useful references are Wigner[4] and Bohr and Mottelson[5].

The Nilsson one body interaction[6] consists of a spin-orbit term, an $L^{2}$ term (to make up 
for the deficiency of the oscillator radial shape) and most important a deformed potential $V(r)=1 / 2 m \omega^{2} r^{2}\left(1-4 / 3 \delta P_{2}(\cos (\vartheta))\right.$. As the deformation parameter $\delta$ aproaches zero we go towards the weak deformation limit. For very large $\delta$ we come to the asymptotic limit where the angle-spin part of the wave function decouples to the form $Y_{L, \Lambda} \chi_{1 / 2, \Sigma}$. For finite $\delta$ the spin-orbit interaction prevents $\Lambda$ and $\Sigma$ from being good quantum numbers. One gets a sum over various $\Lambda$ and $\Sigma$ with the constraint that $\Lambda+\Sigma=K$. Here the formula for the laboratory magnetic moment in the rotational model using the notation of Bohr and Mottelson[7].

$$
\mu=g_{R} J+\left(g_{K}-g_{R}\right) K^{2} /(J+1)\left(1+\delta_{K, 1 / 2}(2 I+1)(-1)^{J+1} b\right)
$$

where

$$
\left(g_{K}-g_{R}\right) b=\left\langle K\left(g_{L}-g_{R}\right) L_{+} \bar{K}\right\rangle+\left\langle K\left(g_{S}-g_{R}\right) S_{+} \bar{K}\right\rangle
$$

and $|\bar{K}\rangle$ is the time reverse of the state $|K\rangle$. Since $g_{R}$ is $Z / A$, for mirror pairs the summed $g_{R}$ is one. Hence, if $K$ is not equal to $1 / 2$ we obtain

$$
2 \times \mu(I S)=J+\left(K g_{K}-K\right) \times K /(J+1)
$$

where $K g_{K}=\left\langle g_{L} L_{z}+g_{S} S_{z}\right\rangle$ evaluated in the intrinsic state. Here $g_{L}$ is also one and $g_{S}=2\left(\mu_{p}+\mu_{n}\right)=1.760$. Keeping in mind $A=9$ and $A=21$ let us consider intrinsic states in the weak deformation limit $p_{3 / 2, K=3 / 2}$ and $d_{5.2, K=5 / 2}$ respectively. We find that

$$
\begin{gathered}
K g_{K}-K=\left(\mu_{p}+\mu_{n}+L-K\right) \\
2 \mu(I S)=J+\left(\mu_{p}+\mu_{n}+L-K\right) \times K /(J+1)
\end{gathered}
$$

where $K g_{K}=\left\langle g_{L} L_{z}+g_{S} S_{z}\right\rangle$ is evaluated in the intrinsic state. When we combine this with the expression at the beginning we obtain

$$
\begin{gathered}
j=L+1 / 2 \quad 2 \times \mu(I S, \text { Schmidt })=L+\mu_{p}+\mu_{n} \\
(\mu(\text { Nilsson })-\mu(\text { Schmidt })) / \mu(\text { Schmidt })=-8.1 \% \text { for } A=9 ;=-3.8 \% \text { for } A=21 .
\end{gathered}
$$
Although the percent changes are rather small the deviations of $\langle\sigma\rangle$ from unity (The Schmidt value) are large. In more detail 
Table I: Experimental values of the spin operator obtained from mirror pairs.

\begin{tabular}{c|c|c|c|c|c}
\hline Mirror Pairs & $J$ & Odd Proton & Odd Neutron & Sum & $\langle\sigma\rangle$ \\
\hline \hline${ }^{9} \mathrm{Li}^{9} \mathrm{C}$ & $3 / 2$ & 3.439 & -1.394 & 2.048 & 1.434 \\
\hline${ }^{21} \mathrm{~F}^{21} \mathrm{Ne}$ & $5 / 2$ & 3.93 & -0.983 & 2.947 & 1.176 \\
\hline${ }^{21} \mathrm{Ne}-{ }^{21} \mathrm{Na}$ & $3 / 2$ & 2.386 & -0.662 & 1.724 & 0.589 \\
\hline${ }^{23} \mathrm{Na}^{23} \mathrm{Mg}$ & $3 / 2$ & 2.218 & -0.536 & 1.681 & 0.479 \\
\hline${ }^{25} \mathrm{Mg}^{25} \mathrm{Al}$ & $5 / 2$ & 3.646 & -0.855 & 2.790 & 0.766 \\
\hline
\end{tabular}

$$
\begin{array}{ll}
A=9: J=3 / 2 \ldots .2 \mu(I S)=1.728 & \langle\sigma\rangle=0.600 \\
A=21: J=5 / 2 \ldots .2 \mu(I S)=2.771 & \langle\sigma\rangle=0.713
\end{array}
$$

Note that for the above states $\langle\sigma\rangle$ is

equal to one in the intrinsic frame but considerably less than one in the lab frame.

We now consider $K=1 / 2$ bands. Since $g_{l}$ and $g_{R}$ are both one the first term in labeled Eq. 9 vanishes and we have

$$
\left(g_{K}-g_{R}\right) b=\left\langle K \mid\left(g_{S}-g_{R}\right) S_{+} \bar{K}\right\rangle
$$

We now list in Table \experimental results for $\langle\sigma\rangle$. The Schmidt

results are given in Table II. We round of all the values to up to three digits beyond the decimal point.

In the single $j$ model for the configurations $\left(d_{5 / 2}\right)^{n}$ the values are as follows:

$$
\begin{array}{lll}
J=3 / 2 & 2 \mu(I S)=3 / 5 * \mu(I S, \text { Schmidt })=1.728 & \langle\sigma\rangle=0.6 \\
& & \\
J=5 / 2 & 2 \mu(I S)=2.880 & \langle\sigma\rangle=1.0
\end{array}
$$

In the weak deformation limit of the Nilsson model one obtains:

$$
J=3 / 2 \quad \psi_{j, K}=d_{5 / 2,3 / 2} \quad 2 \mu(I S)=1.637 \quad\langle\sigma\rangle=0.360
$$


Table II: Isoscalar Schmidt moments

\begin{tabular}{ccc}
\hline & $2 \mu(\mathrm{IS})$ & $\langle\sigma\rangle$ \\
\hline \hline $\mathrm{s}_{1 / 2}$ & 0.880 & 1.000 \\
\hline $\mathrm{p}_{3 / 2}$ & 1.880 & 1.000 \\
\hline $\mathrm{d}_{5 / 2}$ & 2.880 & 1.000 \\
\hline $\mathrm{p}_{1 / 2}$ & 0.373 & $-1 / 3$ \\
\hline $\mathrm{d}_{3 / 2}$ & 1.272 & $-3 / 5$ \\
\hline
\end{tabular}

Table III: Ripka -Zamick Expressions Modified to Yield Isoscalar Magnetic Moments.

\begin{tabular}{lc}
\hline \multicolumn{1}{c}{$\mathrm{p}$ shell } & $2 \mu(\mathrm{IS})$ \\
\hline \hline $\mathrm{J}=\mathrm{K}=1 / 2$ & 0.3733 \\
\hline $\mathrm{J}=\mathrm{K}=3 / 2$ & 1.7320 \\
\hline $\mathrm{s}-\mathrm{d}$ shell & \\
\hline $\mathrm{J}=\mathrm{K}=1 / 2$ & $0.1780 \mathrm{C}^{2}(5 / 2)-0.1746 \mathrm{C}^{2}(3 / 2)+0.3804 \mathrm{C}^{2}(1 / 2)-0.5 \mathrm{C}(5 / 2) \mathrm{C}(3 / 2)+0.5$ \\
\hline $\mathrm{J}=\mathrm{K}=3 / 2$ & $0.1368\left[\mathrm{C}^{2}(5 / 2)-\mathrm{C}^{2}(3 / 2)\right]-0.3645 \mathrm{C}(5 / 2) \mathrm{C}(3 / 2)+1.5$ \\
\hline $\mathrm{J}=\mathrm{K}=5 / 2$ & 2.7720 \\
\hline
\end{tabular}

$$
J=5 / 2 \quad \psi_{j, K}=d_{5 / 2,5 / 2} \quad 2 \mu(I S)=2.771 \quad\langle\sigma\rangle=0.713
$$

Note that the single $j$ and weak deformation Nilsson values are not the same. The first two mirror pairs in Table I have isospin $T=3 / 2$ and the others $T=1 / 2$. The $T=1 / 2$ values of $\langle\sigma\rangle$ are within the single particle limits but this is not the case for $T=3 / 2$. More complete intrinsic wave functions for the cases where $J=K$ have been obtained by Ripka and Zamick [8].They give results for odd proton and odd neutron nuclei from which we can easily infer the isoscalar results. The notation in table III is such that $\mathrm{C}(\mathrm{j})$ is the probability amplitude that the odd particle is in the jj coupling state $n, L, j$.

In Tables $[\mathrm{IV}$ and $\mathrm{V}$ we give a selected list of $2 \mu(\mathrm{IS})$ and $\langle\sigma\rangle$. In Table $\mathrm{V}$ things are rearranged to show the evolution of the expectation of the spin operator from the weak deformation limit to the asymptotic limit.

In the Nilsson model 2 identical particles in the same spacial state have opposite spins so only the odd particle contributes to $\langle\sigma\rangle$ and the value is less than or equal to one.To 
Table IV: Nilsson isoscalar results.

Intrinsic $2 \mu(\mathrm{IS}) \quad\langle\sigma\rangle$

State

\begin{tabular}{|c|c|c|}
\hline \multirow[b]{2}{*}{$\mathrm{p}_{3 / 2,3 / 2}$} & \multicolumn{2}{|c|}{ Weak deformation } \\
\hline & 1.728 & 0.600 \\
\hline $\mathrm{p}_{3 / 2,1 / 2}$ & 1.728 & 0.600 \\
\hline $\mathrm{p}_{1 / 2,1 / 2}$ & 0.3733 & $-1 / 3$ \\
\hline$d_{5 / 2,5 / 2}$ & 2.771 & 0.729 \\
\hline$d_{5 / 2,3 / 2}$ & 2.598 & 0.257 \\
\hline$d_{5 / 2,1 / 2}$ & 2.706 & 0.543 \\
\hline$d_{3 / 2,3 / 2}$ & 1.363 & -0.360 \\
\hline$d_{3 / 2,1 / 2}$ & 1.363 & -0.360 \\
\hline \multirow[t]{2}{*}{$\mathrm{S}_{1 / 2,1 / 2}$} & 0.880 & 1.000 \\
\hline & \multicolumn{2}{|c|}{ Asymptotic $\mathrm{J}=3 / 2$} \\
\hline $\mathrm{Y}_{1,1} \uparrow$ & 1.728 & 0.600 \\
\hline$Y_{1,1} \downarrow$ & 1.424 & -0.200 \\
\hline \multirow[t]{2}{*}{$\mathrm{Y}_{1,0} \uparrow$} & 1.880 & 1.000 \\
\hline & \multicolumn{2}{|c|}{ Asymptotic $\mathrm{J}=1 / 2$} \\
\hline $\mathrm{Y}_{1,1} \downarrow$ & 0.373 & $-1 / 3$ \\
\hline \multirow[t]{2}{*}{$\mathrm{Y}_{1,0} \uparrow$} & 0.880 & 1.0 \\
\hline & \multicolumn{2}{|c|}{ Asymptotic $\mathrm{J}=5 / 2$} \\
\hline $\mathrm{Y}_{2,2} \uparrow$ & 2.771 & 0.729 \\
\hline $\mathrm{Y}_{2,2} \downarrow$ & 2.446 & -0.143 \\
\hline $\mathrm{Y}_{2,1} \uparrow$ & 2.609 & 0.286 \\
\hline \multirow[t]{2}{*}{$\mathrm{Y}_{2,0} \uparrow$} & 2.880 & 1.000 \\
\hline & \multicolumn{2}{|c|}{ Asymptotic $\mathrm{J}=3 / 2$} \\
\hline $\mathrm{Y}_{2,2} \downarrow$ & 1.272 & -0.600 \\
\hline $\mathrm{Y}_{2,1} \uparrow$ & 1.728 & 0.600 \\
\hline $\mathrm{Y}_{2,1} \downarrow$ & 1.424 & -0.200 \\
\hline \multirow[t]{2}{*}{$\mathrm{Y}_{2,0} \uparrow$} & 1.728 & 0.600 \\
\hline & \multicolumn{2}{|c|}{ Asymptotic $\mathrm{J}=1 / 2$} \\
\hline $\mathrm{Y}_{0,0} \uparrow$ & 0.880 & 1.000 \\
\hline
\end{tabular}


Table V: Expectation values of the spin operator for $J=K$.

\begin{tabular}{cccc}
\hline Weak Deformation Limit & \multicolumn{3}{c}{ Asymptotic Limit } \\
\hline \hline & $<\sigma>$ & & $<\sigma>$ \\
\hline $\mathrm{p}_{3 / 2,1 / 2}$ & 0.6 & $\mathrm{Y}_{1,0} \uparrow$ & 1.0 \\
\hline $\mathrm{p}_{3 / 2,3 / 2}$ & 0.6 & $\mathrm{Y}_{1,1} \uparrow$ & 0.6 \\
\hline $\mathrm{p}_{1 / 2,1 / 2}$ & $-1 / 3$ & $\mathrm{Y}_{1,1 \downarrow}$ & -0.2 \\
\hline & & & \\
\hline $\mathrm{d}_{5 / 2,1 / 2}$ & 0.543 & $\mathrm{Y}_{2,0} \uparrow$ & 1.000 \\
\hline $\mathrm{d}_{5 / 2,3 / 2}$ & 0.360 & $\mathrm{Y}_{2,1} \uparrow$ & 0.286 \\
\hline $\mathrm{d}_{5 / 2,5 / 2}$ & 0.729 & $\mathrm{Y}_{2,2} \uparrow$ & 0.729 \\
\hline & & & \\
\hline $\mathrm{d}_{3 / 2,1 / 2}$ & -0.360 & $\mathrm{Y}_{2,0} \uparrow$ & 0.600 \\
\hline $\mathrm{d}_{3 / 2,3 / 2}$ & -0.360 & $\mathrm{Y}_{2,2} \downarrow$ & -0.600 \\
\hline & & & \\
\hline $\mathrm{s}_{1 / 2}$ & 1.000 & $\mathrm{Y}_{2,1 \downarrow}$ & -0.439 \\
\hline
\end{tabular}

obtain values of $\langle\sigma\rangle$ greater than one, components in which the particles are not in the lowest intrinsic states must be introduced. As an example in the weak deformation limit we form the intrinsic state where a particle is promoted from $p_{3 / 2,3 / 2}$ to $p_{1 / 2,-1 / 2}$. Thus the unpaired states are $p_{3 / 2,1 / 2}, p_{3 / 2,3 / 2}$ and $p_{1 / 2,-1 / 2}$. One obtains

$$
2 \mu(I S)=I+K /(I+1) \times\left[\Sigma\left(\left\langle L_{z}\right\rangle+1.760\left\langle S_{z}\right\rangle\right)-K\right]
$$

This is a $K=3 / 2$ band and for $J=3 / 2$ we find that $\left\langle L_{z}\right\rangle=2 / 3,\left\langle S_{z}\right\rangle=5 / 6,2 \mu($ IS $)=1.88$ and $\langle\sigma\rangle=1$. This does not get us what we want. However if we go to the asymptotic limit the unpaired states are $\mathrm{Y}_{1.0} \uparrow \mathrm{Y}_{1,1} \uparrow$ and $\mathrm{Y}_{1,-1} \uparrow$. In this limit we find that $\left\langle L_{z}\right\rangle=0,\left\langle S_{z}\right\rangle=3 / 2$, $2 \mu(I S)=2.164$ and $\langle\sigma\rangle=1.8$. This works.

There are many studies of isoscalar magnetic moments. In the work of Mavromatis et al.[9] it is noted that only with a tensor interaction can one get corrections to the isoscalar momenets of closed major shells plus or minus one nucleon. The systematics of isoscalar moments are discussed in the works of Talmi[10], Zamick|11], B.A. Brown[12], Brown and Wildenthal[13], A.Arima[14], I.Towner[15], and I.Talmi[16]. Closely related to mirror pairs 
are studies of odd-odd $N=Z$ nuclei. It was noted by Yeager et al.[17] that both experimental results and large scale shell model calculations were close to the single $j$ results. To undersand this corrections to Schmidt in first order perturbation theory were performed by Zamick et al.[18]. They found that isoscalar corrections were much smaller than isovector ones for ${ }^{57} \mathrm{Cu}$ and ${ }^{57} \mathrm{Ni}$ mirror pairs. The calculations went in the direction of reducing $\langle\sigma\rangle$. For problems other than this one one will need the supermultiplet quantum numbers of Wigner[4] if one works in the $L S$ coupling basis.

In summary we have shown that the range over which $\langle\sigma\rangle$ can vary is considerably wider than that given by the single particle model. We use the Nilsson model to study this problem and we note some simplicities for the isoscalar mode. The rotational $g$ factor $g_{R}$ gets replaced by one and the expression for a $K=1 / 2$ band simplifies. We show that in this model we can get a value of sigma greater than one only by allowing more than one nucleon to be unpaired. In our example we have three unpaired particles.

The author acknowledges a Morris Belkin visiting professorship at the Weizmann Institute for Spring 2011.He thanks Igal Talmi, Michael Kirson and Michael Hass for their hospitality; also Justin Farischon and Diego Torres for considerable help with the manuscript.

[1] J. Kramer et al. Physics Letters B 678 , 465 (2009)

[2] K. Matsuta et al. Nucl Phys A 588, 153 (1995)

[3] Y.Utsuno Eur. Phys.J. A25, 209 (2005)

[4] E.P. Wigner, Phys. Rev. 51, 106 (1937)

[5] A. Bohr and B.R. Mottelson, Nuclear Structure, V1, New York, W.A. Benjamin, 1969

[6] S.G. Nilsson, Mat. Fys. Medd. Dansk Vid. Selsk. 29, No. 16 (1955)

[7] A. Bohr and B.R. Mottelson, Nuclear Structure, V2, New York, W.A. Benjamin, 1975

[8] G. Ripka and L. Zamick, Physics Letters 23, 347 (1966)

[9] H.A.Mavromatis, L.Zamick and G.E. Brown, Nucl. Phys.80, 541 (1966)

[10] I.Talmi Phys.Rev. 83, 1248 (1951)

[11] L.Zamick, PhysRev. C 15, 824 (1977)

[12] B.A.Brown, J.Phys. G,Nucl. Phys.8, 679 (1982)

[13] B.A.Brown and B.H.Wildenthal,Nucl. Phys. A 474, 296 (1987) 
[14] A.Arima,Hyperfine Interactions 43, 47 (1988)

[15] I.Towner Phys. Rep.155, 264 (1987)

[16] I.Talmi, Hyperfine Excited States of Nuclei (Gordon and Breach,New York) 1971

[17] S.Yeager,S.J.Q.Robinson,L.Zamick and Y.Y.Sharon, EPL 88, 52001 (2009)

[18] L.Zamick,Y.Y.Sharon and S.J.Q. Robinson, Phys.Rev. C82, 067303 (2010) 